\title{
Efficacy and Safety of Elian Granules in Treating Chronic Atrophic Gastritis: Study Protocol For a Randomized, Double-Blind, Placebo-Controlled, Multicenter Clinical Trial
}

\author{
Zhijian Gu ( $\sim$ gogigian@hotmail.com ) \\ Jun Cong \\ Shanghai University of TCM: Shanghai University of Traditional Chinese Medicine \\ Biao Gong \\ Shanghai University of TCM: Shanghai University of Traditional Chinese Medicine \\ Rong Cen \\ Shanghai University of TCM: Shanghai University of Traditional Chinese Medicine \\ Yongqi Chen \\ Shanghai University of TCM: Shanghai University of Traditional Chinese Medicine \\ Qingling Jia \\ Shanghai University of TCM: Shanghai University of Traditional Chinese Medicine \\ Chenheng Wu \\ Shanghai University of TCM: Shanghai University of Traditional Chinese Medicine \\ Jianghong Ling \\ Shanghai University of TCM: Shanghai University of Traditional Chinese Medicine \\ Xudong Tang \\ China Academy of Chinese Medical Sciences
}

Shanghai University of TCM: Shanghai University of Traditional Chinese Medicine https://orcid.org/0000-0002-7965-8280

\section{Research Article}

Keywords: chronic gastritis, chronic atrophic gastritis, Elian granules, traditional Chinese medicine, randomized controlled trial

Posted Date: November 2nd, 2021

DOI: https://doi.org/10.21203/rs.3.rs-740393/v1

License: @ (1) This work is licensed under a Creative Commons Attribution 4.0 International License. Read Full License 


\section{Abstract}

Background: Multifocal atrophic gastritis and intestinal metaplasia are considered to be important links of the gastric precancerous cascade. But, there is a lack of definite therapeutic drugs for them. Many studies have shown traditional Chinese medicine is effective and no serious side effects have been identified. However, the studies that have been carried out were not scientifically rigorous trials. Our aim is to design a high-quality trial for a Chinese patent medicine, Elian granules, to investigate the efficacy and safety of this drug in treating chronic atrophic gastritis patients with or without intestinal metaplasia.

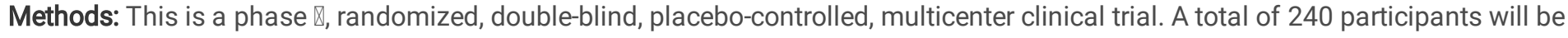
assigned to treatment group or placebo control group with a 1:1 ratio. Then, the experimental drug or placebo will be taken with boiling water, 2 small bags ( $24.2 \mathrm{~g}$ ) each time, twice times a day, half an hour after each meal for 24 weeks. The primary outcome is to observe gastric mucosal histological changes after 6 months in patients with atrophic gastritis with or without intestinal metaplasia based on OLGA/OLGIM. The secondary outcome included dyspepsia symptom score and quality of life scale.

Discussion: This study is designed to evaluate the efficacy and safety of Elian granule in a randomized, double-blind, placebocontrolled, multicenter manner. This trial may not only provide evidence for a phase III clinical trial, but also a vision of an alternative option for chronic atrophic gastritis(CAG) treatment.

Trial registration: The registration number, ChiMCTR2000003929, was assigned by the Registry Platform For Evidence Based Traditional Chinese Medicine on 13 September 2020.

\section{Introduction}

\section{Background and rationale $\{6 a\}$}

Despite the declining incidence of gastric cancer, it remains the most common and highly lethal malignant tumors worldwide, especially in Asian countries ${ }^{[1]}$. This is closely related to the high prevalence of Helicobacter pylori (H. pylori) infection in Asia ${ }^{[2]}$.At present, the association between intestinal metaplasia and non-cardiac gastric cancer and $\mathrm{H}$. pylori infection has been confirmed ${ }^{[3]}$. Its pathogenesis is the gastric precancerous cascade ${ }^{[4]}$ : normal gastric mucosa $\rightarrow$ superficial gastritis (later renamed non-atrophic gastritis, NAG) $\rightarrow$ MAG (multifocal atrophic gastritis)without intestinal metaplasia $\rightarrow$ intestinal metaplasia of the complete (small intestine) type $\rightarrow$ intestinal metaplasia of the incomplete (colonic) type $\rightarrow$ low-grade dysplasia (low-grade noninvasive neoplasia) $\rightarrow$ high-grade dysplasia (high grade noninvasive neoplasia) $\rightarrow$ invasive adenocarcinoma. Studies have shown that the eradication of $\mathrm{H}$. pylori can reduce the incidence of gastric cancer from 63 per 100,000 person-years to 148 per 100,000 person-years in the commonly infected population, which is close to the incidence of 1127 per 100,000 person-years in the non-infected population. However, in the severe atrophic gastritis population, the incidence of gastric cancer in the uninfected and eradicated patients was still 75.9 per 100,000 person years and 1.163 per 100,000 person years, respectively ${ }^{[5]}$. In patients with intestinal metaplasia or dysplasia, eradication of $\mathrm{H}$. pylori does not reduce the risk of gastric cancer ${ }^{[6-7]}$. Therefore, Correa et al. believe that multifocal atrophic gastritis is the true first step in the gastric precancerous cascade[4]. But, there is a lack of definite therapeutic drugs for atrophic gastritis and intestinal metaplasia. COX inhibitors, Nonsteroidal anti-inflammatory drugs (NSAIDs), Rebamipite, moluodan, and antioxidant vitamins have been suggested to be potentially effective against atrophic gastritis and intestinal metaplasia, but the quality of evidence is low. Currently, eradication of $\mathrm{H}$. pylori and endoscopic monitoring are the only relatively clear coping strategies, which are cost-effective $e^{[6-7]}$.

With the widespread application of gastroscopy and pepsinogen, more and more studies have shown that Chinese herbal medicine and Chinese patent medicine may be effective for atrophic gastritis and intestinal metaplasia, but the overall research quality is low. Elian Granule is a Chinese patent medicine in our hospital, which has been used in clinic for more than 20 years.It is composed of zedoary turmeric, coptis, codonopsis, salvia, angelica, dandelion, oldenlandia diffusa, rhizoma atractylodis, raw licorice, pinellia, tangerine peel,tuckahoe, and has the effects of clearing heat, promoting blood circulation and invigorating the spleen. Our preliminary study showed that Elian granule can not only improve the clinical symptoms of patients with atrophic gastritis with or without intestinal metaplasia, but also may relieve gastric mucosa atrophy or intestinal metaplasia in some patients ${ }^{[8-9]}$. The results of animal experiments showed that Elian granule could reverse the heterogeneity of gastric mucosal epithelial cells and reduce the DNA content and polyploid rate of model rats. This reversal may be achieved by promoting the expression of Fas gene protein and inhibiting the 
expression of $\mathrm{Bcl}-2$ gene protein to induce apoptosis of gastric mucosal epithelial cells and increasing the ratio of superoxide dismutase to malondialdehyde in capacity of gastric mucosa ${ }^{[10-12]}$.

\section{Objectives $\{7\}$}

The purpose of this study is to evaluate the efficacy and safety of Elian Granule in the treatment of chronic atrophic gastritis. The primary outcome was to observe gastric mucosal histological changes after 6 months in patients with atrophic gastritis with or without intestinal metaplasia based on OLGA (operative link for gastritis assessment)/OLGIM (operative link for gastric intestinal metaplasia assessment). The secondary outcome included dyspepsia symptom score and quality of life scale. The safety outcome included blood routine, liver function, renal function, urine routine and electrocardiogram. The safe end point will depend on the number of treatment-related adverse events.

\section{Trial design \{8\}}

This current study is randomized, double-blind, placebo-controlled, multicenter clinical trial. The patient allocation ratio is 1:1.

\section{Methods: Participants, Interventions And Outcomes}

\section{Study setting $\{9\}$}

240 patients will be enrolled from 10 hospitals in 6 provinces in China, including:(1)Shuguang Hospital Affiliated to Shanghai University of Traditional Chinese Medicine, (2)Yueyang Hospital of integrated traditional Chinese and Western Medicine,Shanghai University of Traditional Chinese Medicine,(3)Shanghai Pudong Guangming Hospital of Traditional Chinese Medicine,(4)The First Affiliated Hospital of Guangxi University of Chinese Medicine,(5)Affiliated Hospital of Changchun University of Traditional Chinese Medicine,(6)Affiliated Hospital of Shanxi University of Traditional Chinese Medicine (7)The Second Hospital of Anhui Medical University,(8) The Second Affiliated Hospital of Anhui University of Traditional Chinese Medicine,(9)Traditional Chinese Hospital of Lu 'an,(10)Hangzhou Hospital of Traditional Chinese Medicine, are considered for inclusion if they meet the criteria as defined below.

\section{Eligibility criteria $\{10\}$}

\section{Diagnosis}

Referring to Chinese consensus on Chronic Gastritis (2017, Shanghai) formulated by the Society of Digestive Diseases, Chinese Medical Association[13]. Diagnostic criteria for chronic atrophic gastritis as follows:

\section{Clinical manifestations and physical signs}

Chronic gastritis has no specific clinical manifestations,such as epigastric pain, distention, belching, acid regurgitation, nausea, vomiting and loss of appetite.The presence or absence of dyspepsia and its severity does not significantly correlate with chronic gastritis classification, endoscopic features, or gastric mucosal histopathologic staging.Most patients have no obvious physical signs, and sometimes they may have mild upper abdomen tenderness or discomfort.

\section{Endoscopic and histopathological diagnosis}

Endoscopic findings of chronic atrophic gastritis include mixed red and white shades of mucosa dominated by white mucosa, flat or even absent folding, exposure of part of the mucosal blood vessels, and a possible association with mucosal granules or nodules. The diagnosis of chronic atrophic gastritis includes both endoscopic and pathologic diagnoses, while atrophy diagnosed under the white light endoscope has a low agreement with a pathologic diagnosis. The final diagnosis should be based on the pathologic diagnosis.Endoscopic gastric pattern is according to the Kimura-Takemoto classification(Table 1),based on location of the endoscopic atrophic border(Fig.1).The histopathological diagnosis refers to the pathological diagnosis criteria of chronic gastritis in China and visual analogue scale[13].The severity of atrophic gastritis is according to OLGA and OLGIM[14-15].

Table 1 Kimura-Takemoto classification 


\begin{tabular}{|lll|}
\hline Type & Classification & Definition \\
\hline Closed Type & C-1 & the atrophic findings are not visible in the corpus but only in the antrum \\
\cline { 2 - 3 } & C-2 & parabolically above the angulus, and below the middle of the stomach on the lesser curvature \\
\hline C-3 & parabolically above the middle of the stomach on the lesser curvature \\
\hline Opened Type & $0-1$ & the atrophic border lies between the lesser curvature and anterior wall \\
\hline $0-2$ & the atrophic border lies on the anterior wall \\
\hline $0-3$ & the atrophic border lies between the anterior wall and greater curvature \\
\hline
\end{tabular}

\section{Inclusion criteria}

Patients must meet the following criteria to be eligible for the study:

1. Aged 18-70 years, male or female;

2. Those who meet the diagnostic criteria of chronic atrophic gastritis and the test for Helicobacter pylori is negative. (13C or $14 \mathrm{C}$ urea breath test is required);

3. The pathological stage is OLGA-II/III. Two experts from the Department of Pathology are required for diagnosis (Pathological film reading), whose pathological conclusion should reach an agreement;

4. Voluntarily participating in this clinical trial and signing informed consent.

\section{Exclusion criteria}

If the patients meet any of the following criteria at the screening visit, they will not be eligible for the study:

1. Patients with peptic ulcer, severe dysplasia of the gastric mucosa or pathological diagnosis suspected of malignant transformation;

2. Those who have severe digestive system diseases or used to have abdominal surgery;

3. Pregnant or lactating women;

4. In patients with severe heart and lung diseases, malignant tumor, severe diabetes mellitus and chronic liver and kidney dysfunction, ALT and AST of liver function damage were more than 1.5 times of the upper limit of normal, and renal function damage (BUN > 1.2 times of the upper limit of normal, $\mathrm{Cr}>$ the upper limit of normal);

5. PLT $<1.5$ times of the lower limit of normal;

6. Disabled patients (blind, deaf, dumb, mental disorder, mental disorder, physical disability) as prescribed by law;

7. Alcoholics;

8. Those who are known to be allergic to two or more types of drugs or the ingredients of the drug and have allergic constitution;

9. Patients who participated in other clinical trials within 4 weeks. Patients who have taken Weifuchun, Moluodan and folic acid in within 2 weeks;

10. In the opinion of the investigator, those who will cause loss to follow-up, or not suitable for inclusion.

\section{Rejection criteria}

1. Misdiagnosis.

2. No trial drug was used.

3. Patients do not cooperate or cannot complete follow-up.

\section{Withdrawal criteria}

1. Adverse medical events. 
2. Lack of curative effect.

3. Violation of the trail plan (including poor compliance).

4. Lost follow-up (including quitting voluntarily).

5. Subject withdraws informed consent.

\section{Who will take informed consent? \{26a\}}

Patients with chronic atrophic gastritis confirmed by histopathology will be screened for eligibility to participate in this study based on the abovementioned criteria. After the patient has been assessed as eligible, he/she will receive initial study information. After at least 2 weeks of reflection, patients are invited to meet with the research physician to discuss any remaining questions and sign the informed consent.

\section{Additional consent provisions for collection and use of participant data and biological specimens $\{26 \mathrm{~b}\}$}

No additional consent provisions.

\section{Interventions}

\section{Explanation for the choice of comparators $\{6 \mathrm{~b}\}$}

Because there is no definite drug for the treatment of chronic atrophic gastritis with or without intestinal metaplasia, and in order to objectively evaluate the safety and effectiveness of Elian Granules, placebo will be used in the control group. In order to protect patients, the protocol provides that the investigator may withdraw the patients if the patient's condition changes during the trail and other effective treatments are needed.

\section{Intervention description $\{11$ a $\}$}

Elian granules and placebo are produced by Jiangyin Tianjiang Pharmaceutical Co. Ltd.,China for clinical research only (Approval No.2006338).Placebo is similar to Elian granule in color and smell, but it can't be exactly the same. To make a blind test possible, all of the drugs are concealed in unified,sealed and opaque packages with the same labels that contain the drug name, the approval number of the pill, functions, usage, dosage, storage conditions, expiration dates for use and the manufacturer's name. The drugs are administered by an independent clinical assistant in each center, who takes responsibility for the drug's distribution, storage and return. All drugs were registered in the "drug use record for clinical trials", including the date of release of trial drugs, the name of subjects, the quantity of drugs provided and retrieved. According to the intervention plan, there were 6 medium packages in each large package, and 112 small bags of drugs $(12.1 \mathrm{~g} / \mathrm{bag})$ in each medium package. The patients were randomly divided into Elian granule treatment group and placebo control group. All drugs will be taken with hot water half an hour after each meal, 2 small bags each time, twice a day for 24 weeks. A follow-up visit was arranged at 4,12 and 24 weeks after treatment. After the clinical study, patients will receive routine treatment.

\section{Criteria for discontinuing or modifying allocated interventions $\{11 \mathrm{~b}\}$}

Patients can leave the study at any time for any reason if they wish to do so without any consequences. The patient's participation in this study can also be ended by the investigator if the patient is uncooperative and/or does not attend study visits. The patient data that have been collected up to that moment will be included in the analysis. This study will be prematurely ended in case of any abundance in adverse events. Criteria for study termination include any suspected unexpected serious adverse reaction (SUSAR) or serious adverse event (SAE) based on an allergic reaction and clear allergic or iatrogenic effects in two or more patients.

\section{Strategies to improve adherence to interventions $\{11 \mathrm{c}\}$}

During the clinical trial, in order to ensure the compliance of the subjects, the subjects should fully understand the significance of the trial and the importance of taking medicine on time and follow-up. The subjects were required to take medicine according to the regulations and fill in the patient diary card on time. By drug counting method (medication compliance $=$ (actual dosage $/$ required dosage) $\times 100 \%$ ) combined with inquiry to judge the compliance of subjects taking the test drug. Explain patiently to the subjects and ask them to take medicine and follow up as required 
In addition to the prescribed medication, the following traditional Chinese and Western medicine and other therapies that have therapeutic effect on chronic atrophic gastritis are prohibited during the trial period. Once he / she is found to be taking illegal drugs, he / she will withdraw from the study.

1. It is not allowed to use other drugs, such as folic acid, selenium yeast, vitamin $\mathrm{C}$, e, etc.

2. It is not allowed to use Chinese patent medicines that may have certain curative effect on reversing and slowing down the progress of atrophy, such as Weifuchun, Jinghuaweikang capsules, moluodan, Dalitong granules, Qizhiweitong granules, bilingweitong granules, Wenweishu capsules, Xiaojianzhong capsules, Yangweishu capsules, Zhizhukuanzhong capsules, Weisu granules, etc; And any Chinese herbal decoction.

3. It is not allowed to use any acupuncture therapy for the purpose of treating chronic atrophic gastritis during the trial.

The drugs or other treatments that must be taken continuously for complicated diseases can be used under the guidance of researchers. Any drugs used during the trial must be recorded and explained in detail in the study cases, including the name of the disease, drugs, dosage, usage, etc., so that they can be analyzed and reported when summarized.

\section{Provisions for post-trial care $\{30\}$}

The sponsor shall bear the cost of treatment and corresponding economic compensation for the subjects who suffer from the damage or death related to the trial. The sponsor should provide legal and economic guarantee to the researcher, except for those caused by medical malpractice.

\section{Outcomes $\{12\}$}

\section{Primary outcome}

Histological biopsy of gastric mucosa is the primary outcome of this study.OLGA and OLGIM staging were used for pathological evaluation. Evaluation was performed before administration and after $24 \mathrm{~W}$ of administration respectively. The histopathological changes of gastric mucosa were compared between the two groups before and after $24 \mathrm{~W}$ treatment.

\section{Secondary outcomes}

\section{Endoscopic examination}

According to Kimura-Takemoto classification, the gastric atrophy before and after treatment was compared.

\section{Dyspepsia symptom}

Symptom score system was used to evaluate the improvement of dyspepsia (Table 2).In the symptom score system, the score of symptoms will be determined according to the severity, duration and frequency of symptoms. The symptom scores were recorded before administration, $4 \mathrm{~W}, 12 \mathrm{~W}$ and $24 \mathrm{~W}$ respectively. The score of each time will be determined by the sum of all symptom scores. The dyspepsia symptom scores and the changes of each index value before and after treatment were compared between groups. Treatment effect index $(\mathrm{TEI})=($ pre-treatment integral - post-treatment integral) $/$ pre-treatment integral $\times 100 \%$. The curative effect classification was as follows: TEl $\geq 90 \%$ indicates clinically cured, $90 \%>\mathrm{TEI} \geq 70 \%$ indicates remarkable effective, $70 \%>\mathrm{TEI} \geq 30 \%$ indicates effective, $\mathrm{TEI}<30 \%$ indicates invalid.

Table 2 The symptom score system for chronic atrophic gastritis 


\begin{tabular}{|c|c|c|c|}
\hline Symptom & Score & Degree & Grading system \\
\hline \multirow[t]{5}{*}{ Epigastric pain } & 1 & None & - \\
\hline & 2 & Mild & $<2$ times a week \\
\hline & 3 & Moderate & $\geq 3$ times a week, not every day \\
\hline & 4 & Severe & Every day, intermittently \\
\hline & 5 & Extreme severe & Every day, almost continuously \\
\hline \multirow[t]{5}{*}{ Epigastric distention } & 1 & None & - \\
\hline & 2 & Mild & $<2$ times a week \\
\hline & 3 & Moderate & $\geq 3$ times a week, not every day \\
\hline & 4 & Severe & Every day, intermittently \\
\hline & 5 & Extreme severe & Every day, almost continuously \\
\hline \multirow[t]{5}{*}{ Epigastric discomfort } & 1 & None & - \\
\hline & 2 & Mild & $<2$ times a week \\
\hline & 3 & Moderate & $\geq 3$ times a week, not every day \\
\hline & 4 & Severe & Every day, intermittently \\
\hline & 5 & Extreme severe & Every day, almost continuously \\
\hline \multirow[t]{5}{*}{ Early satiety } & 1 & None & - \\
\hline & 2 & Mild & $<2$ times a week \\
\hline & 3 & Moderate & $\geq 3$ times a week, not every day \\
\hline & 4 & Severe & Every day, intermittently \\
\hline & 5 & Extreme severe & Every day, almost continuously \\
\hline \multirow[t]{5}{*}{ Heartburn } & 1 & None & - \\
\hline & 2 & Mild & $<2$ times a week \\
\hline & 3 & Moderate & $\geq 3$ times a week, not every day \\
\hline & 4 & Severe & Every day, intermittently \\
\hline & 5 & Extreme severe & Every day, almost continuously \\
\hline \multirow[t]{5}{*}{ Acid regurgitation } & 1 & None & - \\
\hline & 2 & Mild & $<2$ times a week \\
\hline & 3 & Moderate & $\geq 3$ times a week, not every day \\
\hline & 4 & Severe & Every day, intermittently \\
\hline & 5 & Extreme severe & Every day, almost continuously \\
\hline \multirow[t]{5}{*}{ Belching } & 1 & None & - \\
\hline & 2 & Mild & $<2$ times a week \\
\hline & 3 & Moderate & $\geq 3$ times a week, not every day \\
\hline & 4 & Severe & Every day, intermittently \\
\hline & 5 & Extreme severe & Every day, almost continuously \\
\hline Abdominal discomfort & 1 & None & - \\
\hline
\end{tabular}

Page 7/19 


\begin{tabular}{|l|lll|}
\hline 2 & Mild & $<2$ times a week \\
\hline 3 & Moderate & $\geq 3$ times a week, not every day \\
& 4 & Severe & Every day, intermittently \\
& 5 & Extreme severe & Every day, almost continuously \\
\hline Total symptom score & Equal to the sum of the above scores
\end{tabular}

\section{Quality of Life}

The quality of life was assessed by 12-item short-form health survey(SF-12)(Table 3). The scale items include eight dimensions: General Health $(\mathrm{GH})$, physical functioning (PF), role physical (RP), body pain (BP), role emotional (RE), mental health (MH), vitality (Vt) and social function (SF), and the score is calculated by percentage. The first four dimensions belong to the physical component summary (PCS) level, while the last four dimensions belong to the mental component summary (MCS) level. We will compare the quality of life and the value of each index between the two groups.

Table 3 12-item short-form health survey (SF-12) 


\section{Items}

\begin{tabular}{|ll|}
\hline Score & Grading system \\
\hline 1 & Excellent \\
\hline 2 & Very good \\
\hline 3 & Good \\
4 & Fair \\
5 & Poor \\
\hline
\end{tabular}

In general, would you say your health is?

The following two questions are about activities you might do during a typical day. Does your health now limit you in these activities? If so, how much?

Moderate activities, such as moving a table, pushing a vacuum cleaner, bowling, or playing golf 11 Yes, Limited A Lot ?

\begin{tabular}{|c|c|c|}
\hline & 2 & Yes, Limited A Little \\
\hline & 3 & No, Not Limited At All \\
\hline \multirow[t]{3}{*}{ Climbing several flights of stairs? } & 1 & Yes, Limited A Lot \\
\hline & 2 & Yes, Limited A Little \\
\hline & 3 & No, Not Limited At All \\
\hline
\end{tabular}

During the past 4 weeks have you had any of the following problems with your work or other regular activities as a result of your physical health?

Accomplished less than you would like?

Were limited in the kind of work or other activities?

\begin{tabular}{ll}
1 & Yes \\
\hline 2 & No \\
1 & Yes \\
\hline 2 & No
\end{tabular}

During the past 4weeks, were you limited in the kind of work you do or other regular activities as a result of any emotional problems (such as feeling depressed or anxious)?

Accomplished less than you would like?

Didn't do work or other activities as caefully as usual?

During the past 4weeks, how much did pain interfere with your normal work (including both work outside the home and housework)?

$\begin{array}{ll}1 & \text { Yes } \\ 2 & \text { No } \\ 1 & \text { Yes } \\ 2 & \text { No }\end{array}$

$1 \quad$ Not at all

\begin{tabular}{cl}
2 & A little bit \\
\hline 3 & Moderately \\
4 & Quite a bit \\
\hline 5 & Extremely
\end{tabular}

The next three questions are about how you feel and how things have been during the past 4 weeks. For each question, please give the one answer that comes closest to the way you have been feeling. How much of the time during the past 4 weeks?

Have you felt calm and peaceful?

$\begin{array}{ll}1 & \text { All of the time } \\ 2 & \text { Most of the time } \\ 3 & \begin{array}{l}\text { A good bit of the } \\ \text { time }\end{array} \\ 4 & \text { Some of the time } \\ 5 & \text { A little of the time }\end{array}$




\begin{tabular}{|c|c|c|}
\hline & 6 & None of the time \\
\hline \multirow[t]{6}{*}{ Did you have a lot of energy? } & 1 & All of the time \\
\hline & 2 & Most of the time \\
\hline & 3 & $\begin{array}{l}\text { A good bit of the } \\
\text { time }\end{array}$ \\
\hline & 4 & Some of the time \\
\hline & 5 & A little of the time \\
\hline & 6 & None of the time \\
\hline \multirow[t]{6}{*}{ Have you felt downhearted and blue? } & 1 & All of the time \\
\hline & 2 & Most of the time \\
\hline & 3 & $\begin{array}{l}\text { A good bit of the } \\
\text { time }\end{array}$ \\
\hline & 4 & Some of the time \\
\hline & 5 & A little of the time \\
\hline & 6 & None of the time \\
\hline \multirow{6}{*}{$\begin{array}{l}\text { During the past } 4 \text { weeks, how much of the time has your physical health or emotional problems } \\
\text { interfered with your social activities (like visiting with friends, relatives, etc.)? }\end{array}$} & 1 & All of the time \\
\hline & 2 & Most of the time \\
\hline & 3 & $\begin{array}{l}\text { A good bit of the } \\
\text { time }\end{array}$ \\
\hline & 4 & Some of the time \\
\hline & 5 & A little of the time \\
\hline & 6 & None of the time \\
\hline
\end{tabular}

\section{Safety outcomes}

We will perform the following tests on all subjects to screen for the administration during the study: physical examination (temperature, respiration, heart rate, blood pressure, height and weight), Gastroscope and biopsy, complete blood cell count, urinalysis, liver function (ALT,AST,alkaline phosphatase (ALP), serum total bilirubin (STB), and y-glutamyl transpeptidase(Y-GT),renal function (Cr, BUN), and glomerular filtration rate (GFR) and electrocardiogram (ECG). In addition, a urine pregnancy test will be carried out for female patients of childbearing age.

\section{Participant timeline $\{13\}$}

Table 4 shows the participant timeline. 


\begin{tabular}{|c|c|c|c|c|c|c|}
\hline \multirow{2}{*}{\multicolumn{2}{|c|}{ Visit in doctor's office }} & \multirow{2}{*}{$\begin{array}{l}\text { Enrollment } \\
\text { Visit } 1\end{array}$} & \multirow{2}{*}{$\begin{array}{l}\text { Baseline } \\
\text { Visit } 2\end{array}$} & \multicolumn{2}{|c|}{ Post-allocation } & \multirow{2}{*}{$\begin{array}{l}\text { Close-out } \\
\text { Visit } 5\end{array}$} \\
\hline & & & & Visit 3 & Visit 4 & \\
\hline Time point & & $-4 W-0$ day & Oday & $4 \mathrm{~W} \pm 3$ day & $12 W \pm 3$ day & $24 W \pm 3$ day \\
\hline \multicolumn{7}{|c|}{ Information collection } \\
\hline \multicolumn{2}{|c|}{ Demographic information } & $x$ & & & & \\
\hline \multicolumn{2}{|c|}{ Physical examination } & $x$ & & $x$ & $x$ & $x$ \\
\hline \multicolumn{2}{|c|}{ Written informed consent } & $x$ & & & & \\
\hline \multicolumn{2}{|c|}{ Medical history } & $x$ & & & & \\
\hline \multicolumn{2}{|l|}{ Past history } & $x$ & & & & \\
\hline \multicolumn{2}{|c|}{ Concomitant treatment } & $x$ & & $x$ & $x$ & $x$ \\
\hline \multicolumn{2}{|c|}{ Review Selection } & $x$ & & & & \\
\hline \multicolumn{2}{|l|}{ Allocation } & & $x$ & & & \\
\hline \multicolumn{7}{|l|}{ Interventions } \\
\hline \multicolumn{2}{|c|}{ Treatment group } & & & $x$ & $x$ & $x$ \\
\hline \multicolumn{2}{|c|}{ Control group } & & & $x$ & $x$ & $x$ \\
\hline \multicolumn{7}{|l|}{ Assessments } \\
\hline \multicolumn{2}{|c|}{$\begin{array}{l}\text { Clinical sign and symptom } \\
\text { score }\end{array}$} & $x$ & & $x$ & $x$ & $x$ \\
\hline \multicolumn{2}{|c|}{ Chinese medicine diagnostic } & $x$ & & & & \\
\hline \multirow[t]{2}{*}{ Endoscope } & Kimura-Takemoto classification & $x$ & & & & $x$ \\
\hline & Biospy & $x$ & & & & $x$ \\
\hline \multicolumn{2}{|c|}{$13 \mathrm{C}$ or $14 \mathrm{C}$ breath test } & $x$ & & & & \\
\hline \multicolumn{2}{|l|}{ SF-12 } & $x$ & & & & $x$ \\
\hline \multicolumn{2}{|c|}{ Complete blood cell count } & $x$ & & & & $x$ \\
\hline \multicolumn{2}{|c|}{ Liver function } & $x$ & & & & $x$ \\
\hline \multicolumn{2}{|c|}{ Renal function } & $x$ & & & & $x$ \\
\hline \multicolumn{2}{|l|}{ Urinalysis } & $x$ & & & & $x$ \\
\hline \multicolumn{2}{|l|}{ ECG } & $x$ & & & & $x$ \\
\hline \multicolumn{2}{|c|}{ Urine pregnancy test } & $x$ & & & & \\
\hline \multicolumn{2}{|c|}{ Adverse event record } & & & $x$ & $x$ & $x$ \\
\hline
\end{tabular}


Others

Drug distribution

$x$

$x$

$\times$

Drug recycling and count

$\times$

$\times$

$\times$

Patient Record Card Release

$\times$

Patient Record Card recycling

$\times$

Check patient record card and conduct clinical evaluation

$\times$

$\times$

$\times$

Test End summary

$x$

\section{Sample size $\{14\}$}

A randomized, double-blind, double dummy, parallel controlled, multicenter clinical trial was designed. The sample ratio of the treatment group and the control group was 1:1. Based on the preliminary results of clinical research, the treatment effect of Elian Granule on chronic atrophic gastritis was $85 \%, 63.3 \%$ of the therapeutic effect of Weifuchun was taken as the placebo effect. Using a superiority test, no fewer than 95 subjects should be enrolled based on $a=0.05, \beta=0.10$ and power $=0.90$ in each group. Allowing for a $20 \%$ drop-out, we therefore propose the recruitment of a total sample size of 220 patients. Referring to the phase II clinical trial, the planned total sample size is 240 cases in two groups. In the competitive way, each center should undertake no less than 20 cases.

\section{Recruitment \{15\}}

This study will be recruited in 9 tertiary hospitals and 1 secondary hospital in 6 provinces and cities of China, which can ensure that enough subjects are enrolled.

\section{Assignment of interventions: allocation \\ Sequence generation $\{16 a\}$}

According to the block randomization, using SAS statistical software, given the number of seeds, 240 subjects were randomly arranged, corresponding to 001-240.The case distribution table of each center is as follows (Table 5).

Table 5 Case distribution table of each center 


\begin{tabular}{|llcc|}
\hline $\begin{array}{l}\text { Center } \\
\text { Number }\end{array}$ & Center Name & $\begin{array}{c}\text { Number of } \\
\text { cases }\end{array}$ & $\begin{array}{c}\text { Drug } \\
\text { Number }\end{array}$ \\
\hline 01 & Shuguang Hospital Affiliated to Shanghai University of Traditional Chinese Medicine & $\geq 22$ & $001-022$ \\
\hline 02 & $\begin{array}{l}\text { Yueyang Hospital of integrated traditional Chinese and Western Medicine,Shanghai } \\
\text { University of Traditional Chinese Medicine }\end{array}$ & $\geq 22$ & $023-044$ \\
\hline 03 & Shanghai Pudong Guangming Hospital of Traditional Chinese Medicine & $\geq 22$ & $045-066$ \\
\hline 04 & The First Affiliated Hospital of Guangxi University of Chinese Medicine & $\geq 22$ & $067-088$ \\
\hline 05 & Affiliated Hospital of Changchun University of Traditional Chinese Medicine & $\geq 22$ & $089-110$ \\
\hline 06 & Affiliated Hospital of Shanxi University of Traditional Chinese Medicine & $\geq 22$ & $111-132$ \\
\hline 07 & The Second Hospital of Anhui Medical University & $\geq 22$ & $133-154$ \\
\hline 08 & The Second Affiliated Hospital of Anhui University of Traditional Chinese Medicine & $\geq 22$ & $155-176$ \\
\hline 09 & Traditional Chinese Hospital of Lu 'an & $\geq 22$ & $177-198$ \\
\hline 10 & Hangzhou Hospital of Traditional Chinese Medicine & 20 & $221-240$ \\
\hline
\end{tabular}

\section{Concealment mechanism $\{16 \mathrm{~b}\}$}

The random number generated according to the block randomization is sealed as confidential data. According to the random number, the drugs were blinded by the personnel unrelated to the trial, and the clinical research centers used the drugs in turn according to the assigned drug number and the order of case selection. The blind code is in triplicate and sealed in the office of national drug clinical trial institution of Shuguang Hospital Affiliated to Shanghai University of traditional Chinese medicine, office of clinical trial institution of cooperation unit and statistical unit. Each coded trial drug has corresponding emergency letters, so as to break the blind use in case of emergency. The emergency letters are stored in each trial unit and collected at the end of the trial.

\section{Implementation \{16c\}}

The allocation sequence will be generated by a statistician independent of the data management statistical analysis performed in this trial. The recruitment and intervention of subjects will be completed by the researchers of this project in each clinical research center.

\section{Assignment of interventions: Blinding}

\section{Who will be blinded $\{17$ a $\}$}

Subjects, researchers and data analysts will be blinded.

\section{Procedure for unblinding if needed $\{17 b\}$}

Each drug number is provided with an emergency letter, in which the corresponding drug group is sealed. In case of medical emergency (such as the subject needs rescue, etc.), it can be opened.if it is opened, the letter should be signed and dated by the reader, as well as the reason for opening. In case of emergency, researchers and project leaders should attend, and record the reason, time and place of unblinding in detail, and sign. Inform the clinical trial team leader and clinical supervisor in time after unblinding. The case data should be kept intact. The emergency letter was sent to each clinical research unit with the corresponding drug number, and was taken back after the trial.

\section{Data collection and management}

Plans for assessment and collection of outcomes $\{18 \mathrm{a}\}$ 
This project uses electronic data capture (EDC) to collect and manage data, and redcap system to input data synchronously. Before data entry, the data administrator should conduct EDC and redcap system training to understand the general situation, and master the data entry and export methods. The data manager and the main researchers will work together to write the relevant documents, and the data manager will build and test the electronic case report form (eCFR). The supervisor supervises whether the trial follows the trial plan, confirms that all data are entered synchronously, filled in correctly and completely, and consistent with the original data. If there are errors and omissions, ask the researcher to correct them in time. Only researchers and supervisors can access the subjects' medical records, and they will sign a confidentiality agreement. Data managers, researchers, supervisors and statisticians will conduct blind review and prepare a report on the quality of data management. After the blind review and confirmation of the accuracy of the database, the database will be locked. After the data were locked, they were handed over to the statisticians, and the SAS 6.12 statistical analysis software was used for statistical analysis in a semi-blind way, and the statistical analysis report was written. After analysis, the medical records of the subjects will be kept in the data files of the drug clinical trial institution.

\section{Plans to promote participant retention and complete follow-up $\{18 \mathrm{~b}\}$}

The patients will receive extensive information about the study set-up and requirements during the recruitment. The importance of completion of the follow-up will be stressed. Patients are allowed to stop at any time during the study and are not obliged to give a reason to discontinue. All patients are reminded throughout the study to fill out the questionnaires during study visits. Throughout the follow-up period, the researchers will check responses and if necessary contact patients for completion of their follow-up.

\section{Data management $\{19\}$}

eCRF meeting GCP criteria will be used to collect patient data. The data entered through redcap system will be stored in the research folder on the protected research server and backed up regularly (once every three months).Il the research data (including informed consent) were filled in, reviewed and signed by the supervisor and the person in charge of each unit according to the data management requirements of this protocol. After the agency office sealed, the original copy together with the original medical records were submitted to the experimental unit for retention, one to the data management and statistical analysis unit, and one to Shuguang Hospital Affiliated to Shanghai University of traditional Chinese medicine. The researcher keeps all the research data, including the confirmation of all the participants (can effectively check different records, such as the original records of the hospital), all the original informed consents signed by the patients, all the research medical records, detailed records of drug distribution, etc. Shuguang Hospital Affiliated to Shanghai University of traditional Chinese medicine should keep the clinical trial data for 5 years after the termination of clinical trial.

\section{Confidentiality \{27\}}

Research data will be stored using each participant's research identification code. The key to the identification code list is only provided to the research team during the study, and recorded and protected according to the research guidelines after the study is completed. Details of the patient's identity will not be reported in the publication.

\section{Plans for collection, laboratory evaluation and storage of biological specimens for genetic or molecular analysis in this trial/future use $\{33\}$}

The biopsy samples will be kept in the pathology department of the hospital. Blood samples will be kept in the hospital laboratory. All samples were preserved and destroyed according to the relevant hospital regulations.

\section{Statistical methods}

\section{Statistical methods for primary and secondary outcomes $\{20$ a $\}$}

Full analysis set (FAS): it refers to the ideal subject set that is as close as possible to the principle of intention to analyze (including all subjects who are randomized into the group and receive at least one treatment).For the estimation of the missing value of major variables, if the case data of all treatment processes cannot be observed, carry forward is used to the missing data of the test, and the number of subjects who evaluate the efficacy at the end of each group is consistent with the beginning of the trial.

Per protocol set (PPS): for all patients who meet the experimental treatment protocol, have good compliance, use $80 \%-120 \%$ of the experimental drugs, and complete the CRF requirements, the main variables can be determined, and the baseline variables are not 
missing, and there is no major violation of the experimental protocol.

Safety analysis set (SS): all subjects who received at least one treatment after randomization.

The main variables and comprehensive efficacy analysis were the full analysis set and the per protocol set; Demographic and other baseline characteristics were analyzed using the full analysis set; Safety set is selected for safety evaluation.

Baseline equilibrium analysis will be conducted in FAS.

The equilibrium analysis of the basic value aims at the basic demographic characteristics, vital signs, curative effect related indicators, etc. when the patients are enrolled into the group, so as to explain whether the basic situation of the two groups is comparable. Among them, the quantitative indicators (such as age, course of disease, temperature, pulse, respiration, systolic blood pressure, diastolic blood pressure, TCM syndrome score, etc.) of the two groups were listed, including the number of cases, mean, standard deviation, median, maximum and minimum. The two groups were compared by ANOVA / Kruskal Wallis rank sum test; Qualitative indicators (such as nationality, marital status, heart rate, past history, allergy history, single symptom, etc.), frequency and percentage were listed and compared by $\chi^{2}$ test / precise probability method / rank sum test.

The effectiveness analysis will be carried out in Fas and PPS respectively. According to the OLGA /OLGIM staging changes after treatment, the efficacy of CAG was divided into progressive, stable and improved. The number and percentage of cases were calculated according to the effective rate. $\mathrm{CMH}$ chi square test stratified by center was used to compare the two groups, and the $95 \%$ confidence interval of the rate difference between the two groups was calculated. The curative effect of clinical symptoms was calculated according to the number and percentage of cases cured, markedly effective, effective and ineffective respectively. The $\mathrm{CMH}$ chi square test stratified by center was used to compare the two groups. Changes of mucosal histology before and after treatment: describe the changes relative to the baseline after treatment, calculate the number of cases, mean, standard deviation, median, minimum, maximum, and compare them within the group by paired $t$ test / signed rank test, and compare the changes relative to the baseline between the two groups by analysis of variance. Covariance analysis model was used to compare the difference between the two groups after treatment with respect to the baseline. In the model, the baseline was taken as the covariate, and the effects of grouping and center were considered. On the basis of this model, the least squares mean and $95 \%$ confidence interval of the difference between groups were calculated.

The safety analysis will be carried out in SS. Adverse events: according to the occurrence of adverse events, the number and percentage of cases were listed and compared by chi square test / Fisher exact probability method. During the treatment, all adverse events will be classified and coded according to the common term evaluation standard for adverse events (CTCAE 4.0). The number of cases, times and incidence rate will be calculated according to the system organ classification and standard name after coding, and the details will be described in a list. Laboratory examination related to safety: all completed laboratory examination items are listed in the form of cross table before and after treatment (according to clinical significance), and the abnormal examination items after treatment are listed. Vital signs: describe the changes of physical signs (body temperature, heart rhythm, respiration, systolic blood pressure, diastolic blood pressure) before and after treatment, calculate the number of cases, mean, standard deviation, median, minimum and maximum, and compare them with ANOVA, and list the abnormal examination items after treatment.

\section{Interim analyses $\{21 \mathrm{~b}\}$}

There are no interim analyses planned.

\section{Methods for additional analyses (e.g. subgroup analyses) \{20b\}}

There are no subgroup analyses planned.

\section{Methods in analysis to handle protocol non-adherence and any statistical methods to handle missing data $\{20 \mathrm{c}\}$}

The drug counting method combined with inquiry was used to judge the compliance of the subjects taking the test drug. Medication compliance $=($ actual dosage $/$ required dosage $) \times 100 \% .80 \% \otimes 120 \%$ of the patients had good compliance. The study will be evaluated by intention analysis, which can minimize the missing data.

\section{Plans to give access to the full protocol, participant level-data and statistical code $\{31 \mathrm{c}\}$}


The data sets used in the current study can be provided by the corresponding authors on reasonable request.

\section{Oversight and monitoring}

\section{Composition of the coordinating centre and trial steering committee $\{5 \mathrm{~d}\}$}

This is a multicenter study in 14 hospitals. The daily operation of the study consists of the following personnel:

Principle investigator: responsible for the supervision of the experiment and the medical responsibility of the subjects.

Data administrator: responsible for data acquisition, data quality and safety.

Research Coordinator: responsible for trial registration and coordinating the research of each center.

Supervisor: responsible for monitoring the safety status and research progress of subjects.

Assistant investigator: explore potential subjects, obtain informed consent, and ensure follow-up according to the protocol.

The research group meets once a month. There is no trial Steering Committee, stakeholder and public participation group.

\section{Composition of the data monitoring committee, its role and reporting structure $\{21 \mathrm{a}\}$}

Shuguang Hospital Affiliated to Shanghai University of traditional Chinese medicine appointed a third party as a supervisor to supervise the safety status and research progress of the subjects. The supervisor will contact the research center regularly, including visiting the research center. The degree, nature and frequency of the visit depend on the consideration of the research project and / or end point, the research purpose, the complexity of the research design and the speed of the selection of the subjects.

During the contact, the inspector will:

1. Check and evaluate the progress of the study;

2. Review the research data collected;

3. Check the original data;

4. Find problems and propose solutions.

This is to ensure that:

1. The data are reliable, accurate and complete;

2. The safety and rights of the subjects were protected;

The study was conducted in accordance with the currently approved protocol, GCP and all current registration regulations.

\section{Adverse event reporting and harms $\{22\}$}

The safety of subjects is very important during the study period, and every adverse event will be recorded in detail on the adverse event form (AEF).Adverse events (AE) include any new disease, aggravation of original disease and complications related or unrelated to treatment. Any fatal, life-threatening, disabling or serious event that results in hospitalization or long-term hospitalization shall be considered as SAE. All these data should be recorded on AEF with corresponding treatment methods and reported to China food and Drug Administration (CFDA), provincial food and Drug Administration and ethics committee within 24 hours. Adverse events will be reported to other research centers at the same time. The severity of acute events was divided into three grades: mild, moderate and severe. Mild patients will have a small amount of physical discomfort, can not intervene, no effect on the study. For severe subjects, for their own safety, they should withdraw from the study. In the case of SAE, the researchers can confirm the treatment allocation of the subjects through emergency Unblinding. Any AE related to the trial drug will be treated free of charge. In addition, all adverse events should be recorded and tracked in time until they are properly resolved or stable. The abnormal indexes of physical and chemical examination after medication should be reexamined to normal or stable in time.

Frequency and plans for auditing trial conduct $\{23\}$

Page $16 / 19$ 
In order to meet the requirements of GCP and current regulations, Shuguang Hospital Affiliated to Shanghai University of traditional Chinese medicine can audit the quality of this study at its own discretion. This audit / review can be carried out at any time during and after the study. If such an audit / review is to be carried out, the researcher and the research center should agree to allow the auditor / Reviewer direct access to all relevant documents and arrange time to discuss the findings and related issues with the supervisor / reviewer.

\section{Plans for communicating important protocol amendments to relevant parties (e.g. trial participants, ethical committees) \{25\}}

All substantial amendments will be notified to the competent authority. Non-substantial amendments will be recorded and filed. In case amendments concern or affect participants in any way, they are informed about the changes. If needed, additional consent will be requested and registered. Also, online trial registries will be updated accordingly.

\section{Dissemination plans $\{31$ a $\}$}

The results of this study will be fully disclosed in the journal. Both positive and negative results will be reported.

\section{Discussion}

This study is designed to evaluate the efficacy and safety of Elian granule in a randomized, double-blind, placebo-controlled, multicenter manner.This trial may not only provide evidence for a phase III clinical trial, but also a vision of an alternative option for chronic atrophic gastritis(CAG) treatment

\section{Trial status}

Recruiting started in November 2010. The current protocol is version 1.3 of 13-9-2019. Currently (16th of July 2020), we included 81 patients. Patient recruitment is estimated to be completed around November 2022.

\section{Abbreviations}

CAG

chronic atrophic gastritis, NAG:non-atrophic gastritis, MAG:multifocal atrophic gastritis ,OLGA:operative link for gastritis assessment, OLGIM:operative link for gastric intestinal metaplasia assessment, ALT:alanine aminotransferase, AST:aspartate aminotransferase, BUN:blood urea nitrogen, Cr:creatinine, PLT:platelet, SUSAR:suspected unexpected serious adverse reaction, SAE:serious adverse events, TEl:treatment effect index, ALP:alkaline phosphatase, STB:serum total bilirubin,Y-GT:Y-glutamyl transpeptidase, GFR:glomerular filtration rate, ECG:electrocardiogram, EDC:electronic data capture, eCFR:electronic case report form, FAS:full analysis set, PPS:per-protocol set, SS:Safety analysis set, TCM:traditional Chinese medicine, ANOVA:analysis of variance, AE:adverse event, AEF:adverse event form, CFDA:The China Food and Drug Administration.

\section{Declarations}

\section{Acknowledgements}

We thank all the subjects for participating in this trial. Thanks to China center for evidence based Traditional Chinese Medicine and GCP of Shuguang Hospital Affiliated to Shanghai University of traditional Chinese medicine for their support.

\section{Authors' contributions $\{31 b\}$}

The trial will be performed under the careful supervision of Jianghong Ling,who will oversee the trial during the study. Zhijian Gu,Qingling Jia and Chenheng Wu drafted the initial manuscript with equal contribution. Xudong Tang, Biao Gong $\bowtie$ Rong cen and Yongqi Chen contributed to the conception and design of the protocol and revised the manuscript critically for important intellectual content. Jun Cong participated in the design and coordination of the study, as well as recruiting participants. All authors have reviewed and approved the final manuscript.

\section{Funding \{4\}}


The author(s) disclosed receipt of the following financial support for the research, authorship, and/or publication of this article: This work is supported by National Administration of Traditional Chinese Medicine:2019 Project of building evidence based pratice capacity for TCM, China (No.2019XZZX-XH013) and China Academy of Chinese Medical Sciences:2019 Project of Fundamental research expenses for public welfare departments and institutes of the central government(No.2213-042-2) .

\section{Availability of data and materials $\{29\}$}

The datasets used and/or analyzed during the current study will be made available from the corresponding author upon reasonable request.

\section{Ethics approval and consent to participate $\{24\}$}

Ethical approval was assigned by IRB of Shuguang Hospital Affiliated to Shanghai University of traditional Chinese medicine (No.2020-834-41-01). All participating patients will provide written informed consent.

\section{Consent for publication $\{32\}$}

This manuscript does not contain individual personal data from patients.

\section{Competing interests $\{28\}$}

The author(s) declared no potential conflicts of interest with respect to the research, authorship, and/or publication of this article.

\section{Authors' information (optional)}

Department of Gastroenterology, Shuguang Hospital Affiliated to Shanghai University of traditional Chinese Medicine,185 Pu'an Road, Huangpu District, Shanghai,Post code: 200021.

\section{References}

1. Bray F, Ferlay J, Soerjomataram I, Siegel RL, Torre LA, Jemal A. Global cancer statistics 2018: GLOBO- CAN estimates of incidence and mortality worldwide for 36 cancers in 185 countries. CA Cancer J Clin. 2018;68:394-424.

2. Peleteiro B, Bastos A, Ferro A, Lunet N. Prevalence of Helicobacter pylori infection worldwide: a systematic review of studies with national coverage. Dig Dis Sci. 2014;59:1698-709.

3. Hu B, El Hajj N, Sittler S, Lammert N, Barnes R, Meloni-Ehrig A. Gastric cancer: classifification, histology and application of molecular pathology. J Gastrointest Oncol. 2012;3:251-61.

4. Correa P, Piazuelo MB. The gastric precancerous cascade. J Dig Dis. 2012;13:2-9.

5. Brenner $\mathrm{H}$, Schöttker B. Hermann Brenner. Helicobacter pylori infection, chronic atrophic gastritis and risk of stomach and esophagus cancer: Results from the prospective population-based ESTHER cohort study. Int J Cancer. 2020;146(10):2773-83.

6. Chen H-N, Wang Z, Li X. Zong-Guang Zhou. Helicobacter pylori eradication cannot reduce the risk of gastric cancer in patients with intestinal metaplasia and dysplasia: evidence from a meta-analysis. Gastric Cancer. 2016;19(1):166-75.

7. Pedro Pimentel-Nunes,Diogo Libânio,Ricardo Marcos-Pinto,Miguel Areia,Marcis Leja,Gianluca Esposito,Monica Garrido,llze Kikuste,Francis Megraud,Tamara Matysiak-Budnik,Bruno Annibale,Jean-Marc Dumonceau,Rita Barros,Jean-François Fléjou,Fátima Carneiro,Jeanin E. van Hooft, Ernst J. Kuipers,Mario Dinis-Ribeiro.Management of epithelial precancerous conditions and lesions in the stomach (MAPS II): European Society of Gastrointestinal Endoscopy (ESGE), European Helicobacter and Microbiota Study Group (EHMSG), European Society of Pathology (ESP), and Sociedade Portuguesa de Endoscopia Digestiva (SPED) guideline update 2019.Endoscopy.2019;51(4):365-388.

8. Jun C. Liao Ling-jun, Zhu Mei-ping, Ccai Gan, Zhan Zheng-li.Clinical Efficacy of E-Lian Granule in Patients with Chronic Atrophic Gastritis.An Hui Zhong Yi. Yao Da Xue Xue Bao. 2015;34(1):27-30.

9. Gu Zhi-jian, Jiang TRen-yan,L. Li Yi-men, Zhang Zheng-li, Cai Gan."Elian Granules” for the treatment of chronic atrophic gastritis of spleen deficiency and heat stagnation pattern accompanied with intestinal metaplasia: a randomized controlled clinical trial. Shanghai Journal of Traditional Chinese Medicine. 2015;49(4):40-3. 
10. Cai Gan D, Danbo. Lin Jiang.Effect of Leweijian on Pathological Atypism in Rats with Gastric Precancerous Lesions. Zhongguo Zhong Yao Za Zhi. 2000;9(3):145-7.

11. Cai, Gan. Wang Songpo,Dou Danbo, Lin Jiang.Dynamics of Lewei Decoction on Gastric Mucosal Epithelial Cells in Rats with precancerous Lesions. Xin ZhongYi. 2000;32(2):35-7.

12. Shunhua Z, Rutao C, Gan C, Qiuhong Y. Tian Tao.The effect of Leweijian on exprimentally induced dysphasia of gastric epithelial cell apoptosis and regulation gene(Bcl-2,Fas,ICE).ZheJiang Zhong Yi Xue Yuan Xue Bao, 1998; 23(6):45-48.

13. Fang JY, Du YQ, Liu WZ, Ren JL, Li YQ, Chen XY, Lv NH, Chen YX, Lv B. Chinese consensus on chronic gastritis(2017, Shanghai). J Dig Dis. 2018;19(4):182-203.

14. RUGGE M, GENTA RM. Staging and grading of chronic gastritis. Hum Pathol,2005,36(3):228-233.

15. CAPELLE L G, DE VRIES A C, HARINGSMA J, et al. The staging of gastritis with the OLGA system by using intestinal metaplasia as an accurate alternative for atrophic gastritis. Gastrointest Endosc. 2010;71(7):1150-8.

\section{Figures}
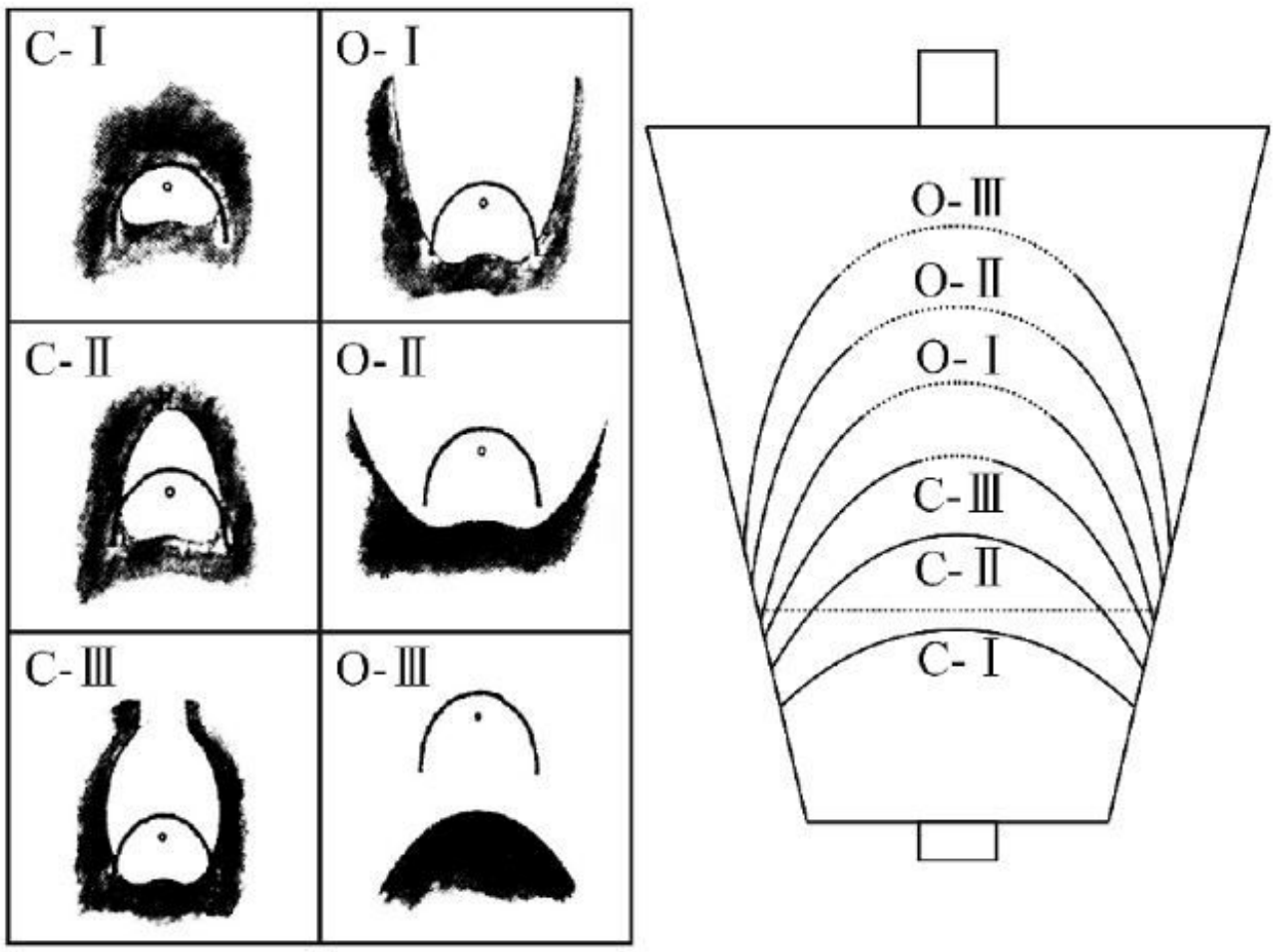

\section{Figure 1}

Extension of the atrophic border and patterns of endoscopic gastric atrophy as classifified by Kimura and Takemoto 